\title{
Managing the Environment for IoT Systems - Toward a "Smart Hospital"
} Eisuke Hanada

Department of Information Science, Faculty of Science and Engineering, Saga University, Saga, Japan

\section{Abstract}

Background: A "smart hospital" is a concept in which various kinds of data can be automatically acquired and analyzed. The acquired data may include patient, staff, device, facility, environmental, and financial data. The Internet of Things (IoT) is a hot topic worldwide, with IoT Systems being introduced to a wide variety of settings, including in the medical field. IoT systems are essential to making a hospital smart. However, the benefits of its use will be not realized if the infrastructure necessary for efficient IoT is not in place.

Methods: The present situation of and problems with the introduction of IoT into hospitals are summarized, with a focus on the electromagnetic environment.

Results: The careful introduction of an IoT system can result in time-savings by the hospital staff, time that can be used for patient treatment and improving the hospital environment, which leads to raising the quality of treatment.

Conclusion: In order to install and make IoT systems work safely and efficiently, managing the environment is the most important consideration, especially for wireless communication. The type and composition of IoT depends on the infrastructure, including the environment.

\section{Introduction}

The concept of "Smart" infrastructure is trending and is being introduced into hospitals. To make a hospital "Smart", data needs to be acquired automatically. To accomplish this, the Internet of Things (IoT) will be an important and essential technology. The target data will be various and include patient, staff, device, facility, and environmental data. IoT already has numerous products that can be connected through the Internet that have been introduced into a variety of settings, including in the medical field [1-3].

Many IoT systems have a structure similar to that shown in Figure 1. As in [4] and [5], ethical problems about collected data, such as privacy, ownership, security, and protocol, are being widely discussed, as are the kinds of data are to be collected and the data format. Although these are important, infrastructure is rarely a focus, but needs to be emphasized. If the infrastructure necessary for realizing efficient IoT is not in place, its potential benefits will be not realized. For example, environmental management of wireless communications and safe introduction should be required before acquisition of information. Also, the electromagnetic environment of sensors should be managed

while transferring data.

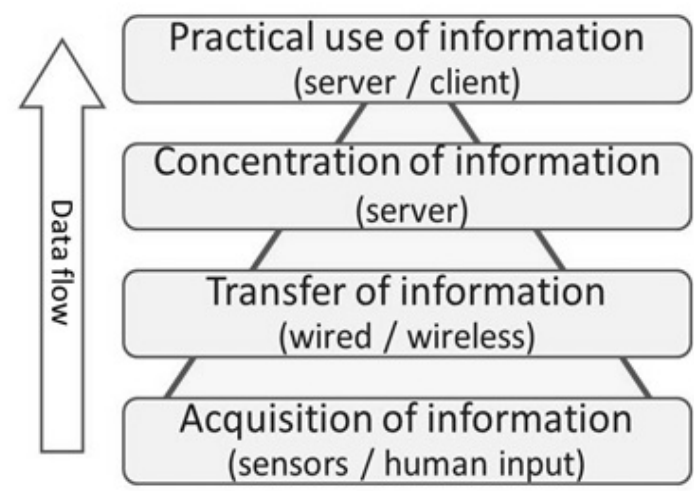

Figure: 1 Structure of an IoT type information system.
Publication History:

Received: June 26, 2018

Accepted: August 24, 2018

Published: August 27, 2018

Keywords:

IoT, Hospital, Wireless

Communicaion, Electromagnetic environment
This paper presents some of the ways that hospital IoT is being used, the conditions of the infrastructure necessary to effective realization of IoT, actual and potential problems, and considerations for the future.

\section{The Demand for and examples of Target Data in Hospital IoT System}

\section{Patient data acquisition}

The aging of the population is rapidly progressing. This has created a situation in which more patients are being treated for chronic disease than are in the acute stage. This means that an increasing number of patients do not require hospitalization. With the introduction of new devices that record biomedical signals in a variety of settings (in hospital, in home, mobile), sensor networks and the introduction of IoT have been proposed as useful support systems for fulfilling such demand.

In hospitals, medical telemetry systems can continuously acquire vital signs and transmit them to a staff station or administrative area. At a terminal in a staff station, staff members can observe multiple patients at the same time. A medical telemetry system connected to a hospital information system (HIS) can be considered IoT.

The continuous acquisition and gathering of information has applications other than those related to the medical practices mentioned above.

${ }^{*}$ Corresponding Author: Prof. Eisuke Hanada, Department of Information Science, Faculty of Science and Engineering, Saga University, Saga, 840-8502, Japan; E-mail: hanada@cc.saga-u.ac.jp

Citation: Hanada E (2018) Managing the Environment for loT Systems - Toward a "Smart Hospital". Int J Comput Softw Eng 3: 134. doi: https://doi. org/10.15344/2456-4451/2018/134

Copyright: (c) 2018 Hanada. This is an open-access article distributed under the terms of the Creative Commons Attribution License, which permits unrestricted use, distribution, and reproduction in any medium, provided the original author and source are credited. 


\section{Data acquisition for raising clinical efficiency and patient safety}

IoT can be realized by adding a communication mechanism to a medical device so that a physician's instructions can be automatically followed or by adding an alarm that can be transmitted to the HIS at the time of a malfunction. In such cases, IoT systems allow medical staff members to quickly understand and react to an unfolding situation by noting the location and having access to real-time data from the device that generated the alarm.

The use of IC tags and sensors that when attached to patients transmit data to the staff has been done experimentally [6,7]. IC tags can be used to prevent patients from wandering or unapproved movement and for early detection of a fall from bed or when walking. The collection of vital signs is also possible by integration with a medical telemetry system. Another benefit is that it will improve efficiency by allowing the staff to understand and quickly react to a wide range of patient behavior [8].

IC tags affixed to the hospital staff have been tested. Their main purpose is to monitor the location and activity of the staff. Currently, most of the targets are nurses, and the technology is used to follow how they go about their tasks as they treat patients and respond to various patient demands.

IC tags attached to medical devices have also been tested. The main purpose of such systems is simply to monitor the location of each device, but some can acquire the operational status of a device, such as if it is switched on or off, the temperature of the device, or by whom the device is used [9].

\section{Environmental data acquisition}

Another target of data acquisition is monitoring the patient environment, such as temperature, humidity, and the illumination of rooms and wards. For the inpatient environment, it is desirable that the temperature and humidity are suited to the disease. The brightness of a room influences both the medical staff's labor environment and the patient's sleep. Continuous monitoring of indoor air quality is necessary for a germfree environment, especially important for patients with an impaired immune system. Air-conditioning management of patient rooms can be done by centralized control, and some rooms have individual air-conditioning. Temperature and humidity control is difficult because of changes in sunshine and visitors coming and going, so it is important to have sensors in each room and if possible for each bed.

In large hospitals, piping is available at each bed, such as for supplying compressed air and oxygen or for aspiration of fluids. Because oxygen leaks can cause explosions and lower the pressure, which reduces the oxygen supply to the patient, the pressure in such piping should always be monitored. With the increase of medical devices driven by electricity, monitoring of the electric power supply to each bed is also required. In addition, it is important to have a monitoring system for the electric power supply in the server room, which is usually not manned.

\section{Financial data acquisition}

Furthermore, hospital accounting systems will be a major target. In Japan, payment for medical treatment is calculated based on the rules of the Japanese government. Hospitals cannot set their own charge for treatment, unless it is done without the use of a national insurance card. Because Japanese medical payment rules are very difficult to understand, the charge is calculated immediately after the treatment is done. Waiting for calculation means that the patient must stay in a reception area together with treated and untreated patients, which increases the risk of infection to and from other patients. The problems with this situation will be ameliorated if an IoT based input system is established.

As stated above, many potential applications for IoT exist in hospitals. The current information collection systems are often not of an IoT style. The realization of and conversion to IoT systems will help hospitals better react to situations where quick judgment is required. In addition, the demand for outpatient data acquisition through IoT systems will continue to grow. This will require a secure network for communication between the hospital and its outpatients. Such a system is complicated by the fact that outpatients patients use various platforms and move freely when outside the hospital.

\section{Technology and its Hazards when Embodying IoT}

At present, many IoT systems use a wireless communications infrastructure, such as ZigBee, Bluetooth, IEEE802.11 series wireless LAN, or terminals, such as those with sensors attached to RFID tags or Raspberry Pi. Wireless communication is especially useful when the object for which information is collected is moving or when there is difficulty connecting the object(s) and the place of collection by cables. It is important to note that there is no optimal communication infrastructure. The fundamental elements for determining the kind of communication infrastructure include the amount of information, the timing or interval of transmission, and the number of terminals and their location.

Especially for wireless communication, care must be taken to control signal propagation and electromagnetic noise. Signals are affected by the building components and furniture in the area where the system is used. Consideration must be taken of the possibility of medical devices as sources of noise. Interference with medical devices by system usage must be carefully considered in medical settings. The main function of some medical devices is to acquire weak biomedical signals, and the surrounding electromagnetic field can affect how well they function [10]. To reduce the effect on medical devices, the electromagnetic field that a system emits should be as weak as possible. This conflicts with the goals of many systems that use strong output to enable communication at long distances.

\section{Electromagnetic Noise Sources and Their Effect}

\section{Sources of electromagnetic noise}

As mentioned above, some medical devices are themselves the source of electromagnetic noise. Electromagnetic noise sources in hospitals include common electricity powered fixtures, signals from objects that are brought into the building, and radio waves or electromagnetic fields generated outside the building that invade it. Examples of problematic items commonly brought into the hospital include mobile phones, Wi-Fi routers, and game machines with a communication facility. Guidelines for the use of these devices should be created for each facility.

Serious problems can occur when the electromagnetic field a medical device emits becomes an electromagnetic noise source for 
other medical devices. Microwave therapy equipment and some heaters emit electromagnetic noise in the same frequency band as the ISM band, which is often used in the communication infrastructure of IoT [11]. Although not specifically a medical device, microwave ovens are often installed in hospital wards. Careful planning of the location of the microwave equipment and careful installation of the IoT system can minimize the electromagnetic effect. Fortunately, few instruments that are possible noise sources use the $300 \mathrm{MHz}$ band, which is used by most active RFID tags.

Some research has been done on electromagnetic field leakage from LED lamps. Recently, electromagnetic field leakage from the circuit board of a LED lamp placed in a ceiling near the antenna of a medical telemetry system was shown to be the cause of radio disturbance [12]. Some LED lamps leak electromagnetic noise in a wide frequency band beyond $300 \mathrm{MHz}$, which is the upper limit of the target frequency band in the regulations of CISPR 15. A major cause of radio disturbance is a lack of consideration during the hospital design phase of the arrangement of the various devices that will be used in a particular setting. This is because the management section responsible for the antenna of a medical telemeter usually differs from the one that manages the lighting. When IoT systems are used, careful consideration must be given to ways to eliminate sources of electromagnetic noise that are too close to instruments with transmission and reception capabilities.

\section{Elements that affect signal propagation}

Elements that affect signal propagation include the components of building construction, such as walls, floors, doors, and windowpanes, and fixtures, such as metal cabinets. The use of chalk walls and reinforced plastic insures permeability to electromagnetic waves. Superficial inspection of the surface of walls and ceilings is insufficient because the inner wall composition may be quite different than the surface. The materials used as building components and their qualities are important to the successful implementation of an IoT system [13]. Another example of building construction that can affect signal propagation is the metal ducts of air conditioning systems, which are often placed in ward ceilings. It is regrettable that the staff of many hospitals and clinics show little concern about these problems. Developers and vendors should be careful to address these problems and to discuss them extensively with the hospital staff.

Wave propagation simulation is useful in the planning of the hospital environment. Unfortunately, because of the computational complexity required for the use of the simulation technology, the location of a signal source is too often set without prior simulation. Another problem is the difficulty of simulating signal propagation from a moving terminal.

\section{Enabling Control of Electromagnetic Fieldes}

If an IoT system uses wireless communication, the electromagnetic environment of the area in which the system is in operation must be well maintained. Proper maintenance of the electromagnetic environment requires not only knowing the building materials but also controlling the electromagnetic fields in the hospital by the use of shielding and absorbing technology.

As mentioned above, building components affect signal propagation. Signal invasion can be prevented by the careful placement of reflectors and absorbers, but there is no need to totally shield the hospital building because useful public communication signals, such as from cellular phones and public $\mathrm{Wi}-\mathrm{Fi}$, will be unable to reach to the terminals inside. From another viewpoint, the information manager might also wish to shield the hospital building in such a way that wireless LAN signals used inside a hospital building will not leak outside.

Band-stop shielding technology is suitable for this condition. For example, non-metal periodic structures are promising for the selective shielding of specific frequencies. It is an application of the band gap characteristic of photonic crystals to microwave regions that change the scale of the structure [14]. This type of shielding technology can control the brightness of a room, which influences both the medical staff's labor environment and the patient's environment. Prof. Kudou has numerically analyzed the shielding effect of a window that consists of hollow metallic square-cylinders and a few sheets of shielding glass and has shown useful data for designing a comfortable MRI room [15].

\section{Proposal for Procedures to Build a Safe and Efficient IoT Environment}

Based on the above, I here propose procedures for the safe introduction of wireless communications and for insuring that they operate at the expected speed. Also, an example in which the proposals were realized is shown. In order for wireless communications to operate with the expected transfer rate, signals from the APs must reach the desired area at the required intensity. For this purpose, the following procedures are necessary.

1. Assessment of signal range based on the hospital floor plan: It is not necessary to make communication possible in all the rooms of a hospital.

2. Acquisition of component information: Information concerning the structure and material of floors, walls, doors, and glass should be acquired.

3. Determination of antenna (AP) location: It would be best to do a simulation using the above-mentioned results and plan the location of APs based on the results.

4. Investigation while under construction: As construction advances, electromagnetic fields that invade from the outside need to be measured, and the number and location of APs changed as necessary. In addition, caution must be taken when determining the location of the cables that provide electricity to the APs.

5. Investigation after completion: After a building is completed and fixtures are in place, signal reach must be tested in all places where communication is to be done. The intensity (transmission rate) of the signals received from the APs must be checked and the output of the APs adjusted as required.

6. Investigation of electromagnetic noise sources and implementation of measures to eliminate their effect: The electromagnetic environment should be investigated soon after the system is put in operation. If electromagnetic noise sources are discovered, elimination or shielding, distance reservation, etc. must be done.

The above procedures must be dealt with when installing wireless LAN, and other elements should be examined (for example, security) [16]. 
Citation: Hanada E (2018) Managing the Environment for IoT Systems - Toward a "Smart Hospital". Int J Comput Softw Eng 3: 134. doi: https://doi. org/10.15344/2456-4451/2018/134

Page 4 of 4

The above procedures were applied to the university hospital at which I previously worked [17]. Below, the example of one floor is shown. This floor is a ward of approximately $38 \mathrm{~m} \mathrm{x} 51 \mathrm{~m}$. At the time of its design, the proposed number of APs was six, based only on the floor plan. However, the following facts became clear as the plan progressed. The floor was made of concrete reinforced with steel rods, the door of each patient room was made of metal, and many pipe spaces that had metal doors were installed in the toilet in each patient room. This created a high possibility that signals would not reach into some patient rooms because the APs were installed in the corridors, which is the common method. In cooperation with the AP vendor, we did an electromagnetic field propagation simulation. The results for this floor showed that the required transmission speed could be obtained in all the required areas if least 12 Aps were installed. In addition, both IEEE802.11g and 802.11a were used in the wireless LAN. In a second simulation after completion of the system, it turned out that the range of access differed for the two standards, so we needed to adjust the output for some of the APs.

\section{Realizatilon of a "Smart Hospital" Using ToI system}

As stated above, there are many kinds of demand for IoT systems in hospitals. Insufficient consideration during preparation and poor management of the environment and infrastructure are the greatest barriers to the effective utilization of IoT systems. Also, it is important to design an infrastructure that suits the various elements required by the individual user, as described in this paper, and to thoughtfully consider the environment and manage it in a way that best suits the characteristics of the infrastructure.

A "smart hospital" is a concept that will be realized in the near future in which many kinds of data can be automatically acquired and analyzed. The analyzed data will be useful information to the staff and patients. IoT systems are essential to making a hospital "smart", but, to install and make them work safely depends on the infrastructure, which must take into account environmental concerns. Managing the environment is the most important consideration, especially for wireless communication.

The introduction of an IoT system can improve efficiency, which will lead to more effective use of the time of the hospital staff. The staff can use the saved time for improved patient care and to improve the hospital environment. This is of great benefit to the patient and leads to raising the quality of treatment.

\section{Acknoweledgment}

This research was supported by JSPS Kakenhi grant No. 15H04794. This paper was written based on the conference paper in IEEE APEMC 2018.

\section{Competing Interests}

The authors declare that they have no competing interests.

\section{References}

1. Gubbia J, Buyyab R, Marusic S, Palaniswami M (2013) Internet of Things (IoT): A vision, architectural elements, and future directions. Future Generation Computer Systems 29: 1645-1660.

2. Lomotey RK, Pry J, Sriramoju S (2017) Wearable loT data stream traceability in a distributed health information system. Pervasive and Mobile Computing 40: 692-707.

Int J Comput Softw Eng

ISSN: 2456-4451
3. Park A, Chang H, Lee KJ (2017) Action Research on Development and Application of Internet of Things Services in Hospital. Healthc Inform Res 23: 25-34.

4. Qi J, Yang P, Min G, Amft O, Dong F, et al. (2017) Advanced internet of things for personalised healthcare systems: A survey. Pervasive and Mobile Computing 41: 132-149.

5. Mittelstadt B (2017) Ethics of the health-related internet of things: a narrative review. Ethics and Information Technology 19: 157-175.

6. Portela F, Miranda F, Santos MF, Abelha A, Machado J, et al. (2017) An Online-Processing Critical Patient Monitoring System- An Interoperability Overview. Computer Science and Information Systems 14: 491-515.

7. Hanada E (2013) Effective Use of RFID in Medicine and General Healthcare. Information Systems and Technology for Organizations in a Networked Society. IGI book Hershey PA USA 335-352.

8. The Ministry of Health, Labour and Welfare (Japan) Establishing the Community-based Integrated Care System. Webpa.

9. Issa, P. Isaas, \& P. Kommers (Ed.) (2013) Information Systems and Technology for Organizations in a Networked Society. IGI book Hershey PA USA.

10. Hanada E, Sumi T, Ishitobi S, Kudou T, et al. (2012) Improving the clinical physiological examination environment - Avoiding EMD on medical equipment used for detecting biological signals. Przeglad Eletrotechniczny.

11. Hanada E, Itoga S, Tamai, Nagae Y, Kurosawa H, (2010) Unnecessary" Electromagnetic Field Radiated From Medical Devices," ISMICT A1-5, Taipei.

12. Ishida K, Arie S, Gotoh K, Hanada R, Hirose M, et al. (2017) Electromagnetic Compatibility of Light-emitting Diode (LED) Lamps and Wireless Medical Telemeters. EMD.

13. Hanada E, Kudou T (2016) Insuring the "network availability" necessary for effective wireless communication in hospitals. Medical Informatics Europe (MIE) 47: 74 .

14. Hanada E, Kudou T (2018) Managing the Electromagnetic Environment of Hospital loT Systems. IEEE APEMC

15. T. Kudou, E. Hanada (2011) FDTD simulations for constructing appropriate medical/healthcare electromagnetic environment. APEMC.

16. Hanada E, Kudou T, Tsumoto S (2013) Installation of Secure, Always Available Wireless LAN Systems as a Component of the Hospital Communication Infrastructure. J Med Syst 37: 9939.

17. Hanada E, Kudou R (2016) Managing the Availability of Hospital Wireless Communication Systems.
This article was originally published in a special issue:

Internet of Things

Handled by Editor:

Dr. Makhoul Abdallah

Department of Computer Science

University of Franche-comté

France

Volume 3. 2018. 134 